\title{
Rice Yield Prediction for Cauvery Delta Zone of Tamil Nadu using Weather Based Statistical Model
}

\author{
G. Senthil Kumar ${ }^{*}$ and K. Subrahmaniyan ${ }^{2}$ \\ ${ }^{1}$ Department of Rice, Tamil Nadu Agricultural University, Coimbatore - 641 003, India \\ ${ }^{2}$ Tamil Nadu Rice Research Institute, Aduthurai, Thanjavur district, Tamil Nadu, India \\ *Corresponding author
}

\section{A B S T R A C T}

\begin{tabular}{|l|}
\hline Ke y w o r d s \\
Rice, Yield, \\
Weather, Forecast, \\
Statistical model, \\
regression
\end{tabular}

Yield forecasting regression models utilise data on yield and weather variables for past several years pertaining to locations under consideration. By studying the relationship of yield with different weather elements, predictors are identified. Generally, rainfall, temperature, humidity, rainy days, dry days and cloud amount etc., during critical phases of crop growth fulfill the criteria to be predictors. The weather variability both within and between seasons is unmanageable source of variability in yield. The weather variables affect the crop differently during various stages of development. Thus, extent of weather influence on crop yield depends not only on the magnitude but also on the distribution pattern of weather variables over the crop season. Statistical method has been performed for forecasting crop yield for Rice crop at Cauvery delta zone of Tamil Nadu. The forecast has been developed by using crop yield data considering four weather variables (Maximum and Minimum temperature, Rainfall, Morning and Evening Relative Humidity) simultaneously. Long term weather (1995-2016) and historical crop yield (1999-2016) data were utilized in the model. District level rice yield forecast for delta districts viz., Thanjavur, Thiruvarur, Nagapattinam, Trichy, Perambalur, Ariyalur and Cuddalore was issued by using the statistical model at mid-season (F2) and pre-harvest stage (F3) during Kharif, 2017 and Rabi, 2017-18.

\section{Introduction}

Variation in crop yield over years has two components. One is more or less systematic rise in yield derived from improved cultivars, better crop management and the interaction between cultivars and management (Boltan, 1981). Weather plays an important role in crop growth as well as crop development. Therefore, models based on weather parameters can provide reliable forecast of crop yield in advance of harvest. A number of yield forecasting models have been developed for various crops.

Yield forecasting regression models utilise data on yield and weather variables for past several years pertaining to locations under consideration (Surrender Paul et al., 2012). By studying the relationship of yield with different weather elements, predictors are identified. 
Generally, rainfall, temperature, humidity, rainy days, dry days and cloud amount etc., during critical phases of crop growth fulfill the criteria to be predictors (Jayantha Sarkar, 2003). Crop yield in different years is affected by technological factors will increase the yield smoothly through time and therefore, year-number can be used to study the overall effect of technology on yield. The weather variability both within and between seasons is unmanageable source of variability in yield. The weather variables affect the crop differently during various stages of development (Tripathi et al., 2012). Thus, extent of weather influence on crop yield depends not only on the magnitude but also on the distribution pattern of weather variables over the crop season.

\section{Materials and Methods}

In Tamil Nadu, district level rice yield Forecast for Cauvery delta zone is more important because this zone is rice bowl of Tamil Nadu. The most commonly adopted cropping sequence in delta region is Kuruvai rice (Kharif) - thaladi rice ( $R a b i)$ - rice fallow black gram/gingelly/cotton In the rice based cropping system, it is either single (Samba: 2.99 lakh ha) or double cropped (Kuruvai: 1.68 lakh ha and Thaladi: 1.44 lakh .ha). Pulses like Black gram (1.43 lakh ha) and green gram (0.46 lakh. ha) are next important crops grown in the rice fallows throughout the delta region from January onwards under no tillage condition. District level rice yield Forecast for delta districts viz., Thanjavur, Thiruvarur, Perambalur, Nagapattinam,Cuddalore, Ariyalur and Trichy was issued by using Statistical Model at midseason (F2) and pre-harvest stage (F3) during Kharif, 2017 and Rabi 2017-18. There is a need of dividing the crop season into different intervals. Thus, a technique based on relatively smaller number of manageable variables and at the same time taking care of entire weather distribution may solve the problem. The regression model which was used for yield forecasting is weather indices based model which is modified Hendricks and Scholl method at IASRI (Agrawal and Mehta, 2007). Statistical method has been performed for forecasting crop yield for rice crop at selected districts. The forecast has been developed by using crop yield data considering the four weather variables (Maximum and Minimum temperature, Rainfall, Morning and Evening Relative Humidity) simultaneously. Long term weather (1995-2015) and historical crop yield (19992015) data were utilized in the model.

Final yield forecast will be given by using statistical regression model using this formula.

$$
Y=A_{0}+\sum_{i=1}^{p} \sum_{j=0}^{1} a_{i j} Z_{i j}+\sum_{i \neq i^{\prime}=1}^{p} \sum_{j=0}^{1} a_{i i{ }^{\prime}, j} Z_{i{ }^{\prime}, j}+c T+e
$$

Where,

$$
\begin{aligned}
Z_{i j} & \sum_{w=1}^{m} r_{i w}^{j} X_{i w} \\
Z_{i i j}{ }^{\prime}= & \sum_{w=1}^{m} r_{i i^{\prime} w}^{j} X_{i w} X_{i w}^{\prime}
\end{aligned}
$$

\section{Results and Discussion}

\section{Rice yield prediction during Kharif, 2017}

District level rice yield forecasting models of rice are presented for 5 districts viz., Thanjavur, Thiruvarur, Trichy, Nagapattinam and Cuddalore districts in Table 1. The weather data for maximum temperature ( $T$ max), minimum temperature ( $\mathrm{T}$ min), relative humidity (RH I \& RH II) and daily rainfall values were collected for the period of 1995 2016. 
Kharif season - mid season forecast (F2)

District level rice yield forecast was prepared and the forecasted yields are given during mid season of Kharif, 2017. Crop yield forecast for rice was given for five selected districts (Thanjavur, Thiruvarur, Trichy, Nagapattinam and Cuddalore) during Kharif season 2016. The highest paddy yield of $4972 \mathrm{~kg} / \mathrm{ha}$ has been predicted for Thiruvarur district while the lowest yield has been predicted for Thanjavur district $(3891 \mathrm{~kg} / \mathrm{ha})$. The major rice varieties grown in Cauvery delta zone during Kharif season is ADT 43, ADT 45 and ADT 36.

\section{Kharif season - pre- harvest forecast (F3)}

District level rice yield forecast was prepared and forecasted yields are given in Table 2. In F3 forecast, higher paddy yield of $4987 \mathrm{~kg} / \mathrm{ha}$ was predicted in Trichy district followed by Thiruvarur district $(4825 \mathrm{~kg} / \mathrm{ha})$. Lower yield of $3556 \mathrm{~kg} / \mathrm{ha}$ was predicted in Thanjavur district.

Table.1 Crop yield forecast of Kharif Rice (F2: Flowering Stage) during $15^{\text {th }}$ September, 2017

\begin{tabular}{|c|c|c|}
\hline Districts & Equation & $\begin{array}{c}\text { Forecast } \\
\text { Yield 2017 (kg/ha) }\end{array}$ \\
\hline Thanjavur & $\mathrm{Y}=-1906.65+2.52(\mathrm{Z} 241)$ & 3891 \\
\hline Thiruvarur & $\mathrm{Y}=2743.51+0.386(\mathrm{Z} 131)$ & 4972 \\
\hline Nagapattinam & $\mathrm{Y}=11002.33+289.54(\mathrm{Z} 11)+0.15(\mathrm{Z} 240)$ & 3079 \\
\hline Trichy & $\mathrm{Y}=2781.830791+3.29(\mathrm{Z} 121)$ & 4969 \\
\hline Cuddalore & $\mathrm{Y}=283.72+1.047(\mathrm{Z} 21)$ & 4951 \\
\hline
\end{tabular}

Table.2 Crop yield forecast of Kharif Rice (F3: Pre-Harvesting Stage) during $15^{\text {th }}$ October, 2017 for Cauvery Delta Zone of Tamil Nadu

\begin{tabular}{|c|c|c|}
\hline District & Equation & $\begin{array}{c}\text { Forecast yield 2017 } \\
\text { (kg/ha) }\end{array}$ \\
\hline Thanjavur & $\mathrm{Y}=3422.209+0.14(\mathrm{Z} 131)$ & 3556 \\
\hline Thiruvarur & $\mathrm{Y}=4119.01+1.96(\mathrm{Z} 141)$ & 4825 \\
\hline Nagapattinam & $\mathrm{Y}=11002.33+289.54(\mathrm{Z} 11)+0.15(\mathrm{Z} 240)$ & 4379 \\
\hline Trichy & $\mathrm{Y}=-1449.89+210.76(\mathrm{Z} 21)$ & 4987 \\
\hline Cuddalore & $\mathrm{Y}=8558.76+23.84(\mathrm{Z} 20)$ & 4435 \\
\hline
\end{tabular}

Table.3 Verification of Kharif, 2017 yield forecast

\begin{tabular}{|c|c|c|c|c|c|}
\hline Districts & Area (ha) & $\begin{array}{c}\text { Predicted yield F3 } \\
\text { (kg/ha) }\end{array}$ & $\begin{array}{c}\text { Actual yield } \\
(\mathbf{k g} / \mathbf{h a})\end{array}$ & $\begin{array}{c}\text { Yield } \\
\text { difference } \\
(\mathbf{k g} / \mathbf{h a})\end{array}$ & $\begin{array}{c}\text { Error } \\
(\mathbf{\%})\end{array}$ \\
\hline Thanjavur & 28298 & 3556 & 3712 & -156 & $\mathbf{- 4 . 2 0}$ \\
\hline Thiruvarur & 18,394 & 4825 & 4489 & 336 & $\mathbf{7 . 4 8}$ \\
\hline Nagapattinam & 22,683 & 4379 & 4012 & 367 & $\mathbf{9 . 1 4}$ \\
\hline Trichy & 3430 & 4987 & 4880 & 107 & $\mathbf{2 . 1 9}$ \\
\hline Cuddalore & $\mathbf{8 3 1 0}$ & $\mathbf{4 4 3 5}$ & $\mathbf{4 9 0 6}$ & $\mathbf{- 4 7 1}$ & $\mathbf{- 9 . 6 0}$ \\
\hline
\end{tabular}


Table.4 Yield Forecasting for Kharif rice during F2 $\left(15^{\text {th }}\right.$, September $) \&$ F3 $\left(15^{\text {th }}\right.$, October $) 2017$ at Cauvery Delta Zone in Tamil Nadu

\begin{tabular}{|c|c|c|c|c|c|c|}
\hline \multicolumn{5}{|c|}{ F2 Forecasting date $15^{\text {th }}$, October 2017} & \multicolumn{2}{|c|}{$\begin{array}{l}\text { F3 Forecasting date } 15^{\text {th }} \text {, October } \\
\qquad 2017\end{array}$} \\
\hline $\begin{array}{l}\text { Sl. } \\
\text { No }\end{array}$ & Districts & $\begin{array}{c}\text { Average } \\
\text { yield } \\
\text { (kg/ha) }\end{array}$ & $\begin{array}{l}\text { Weather } \\
\text { elements }\end{array}$ & Equation & $\begin{array}{l}\text { Weather } \\
\text { element }\end{array}$ & Equation \\
\hline 1 & Thani & 3349 & RH II & $Y=-1906$. & $\mathrm{RH}$ & $Y=3422.209$ \\
\hline 2 & Thiruvarur & 2914 & $\begin{array}{c}\mathrm{Tx} \\
\mathrm{Tx} * \mathrm{RF}\end{array}$ & $Y=2743.51$ & $\mathrm{Tx} * \mathrm{RF}$ & $Y=4119.01$ \\
\hline 3 & Nagapattinam & 2844 & $\mathrm{Tx} * \mathrm{Tn}$ & $\begin{array}{c}\mathrm{Y}=11002.33+289.54(\mathrm{Z} 11)+ \\
0.15(\mathrm{Z} 240)\end{array}$ & $\mathrm{Tx} * \mathrm{Tn}$ & $\begin{array}{c}Y=11002.33+289.54(\mathrm{Z} 11)+ \\
0.15(\mathrm{Z} 240)\end{array}$ \\
\hline 4 & Trichy & 3850 & $\mathrm{Tn}$ & $\mathrm{Y}=2781.830791+3.29(\mathrm{Z} 121)$ & $\operatorname{TnTn} * \mathrm{RF}$ & $Y=-1449.89+210.76(Z 21)$ \\
\hline 5 & Cuddalore & 3425 & $\mathrm{Tn}$ & $\mathrm{Y}=283.72+1.047(\mathrm{Z} 21)$ & $\mathrm{Tn} * \mathrm{RHI}$ & $Y=8558.76+23.84(\mathrm{Z} 20)$ \\
\hline
\end{tabular}

Table.5 Crop Yield Forecast of Rabi Rice (F2: Flowering Stage) during 30 ${ }^{\text {th }}$ December, 2017 for Cauvery Delta Zone of Tamil Nadu

\begin{tabular}{|c|c|c|}
\hline District & Equation & $\begin{array}{c}\text { Forecast yield, 2017 } \\
\text { (kg/ha) }\end{array}$ \\
\hline Thanjavur & $\mathrm{Y}=-309.3+0.8(\mathrm{Z} 151)+2.1(\mathrm{Z} 241)$ & 2553 \\
\hline Thiruvarur & $\mathrm{Y}=-1770+2.695(\mathrm{Z} 241)$ & 2503 \\
\hline Nagapattinam & $\mathrm{Y}=671.68+7.7(\mathrm{Z} 241)$ & 2027 \\
\hline Trichy & $\mathrm{Y}=-2312+0.2(\mathrm{Z} 251)$ & 4556 \\
\hline Karur & $\mathrm{Y}=3212+0.3(\mathrm{Z} 250)$ & 2474 \\
\hline Perambalur & $\mathrm{Y}=26.6+2.6(\mathrm{Z} 121)$ & 4516 \\
\hline Ariyalur & $\mathrm{Y}=-2213+0.2(\mathrm{Z} 250)$ & 3660 \\
\hline Cuddalore & $\mathrm{Y}=-333.9+2.12(\mathrm{Z} 141)+0.3(\mathrm{Z} 250)$ & 3105 \\
\hline
\end{tabular}

Table.6 Crop Yield Forecast of Rabi Rice Crop (F3: Pre-Harvesting Stage) during 30 ${ }^{\text {th }}$ January 2018 for Cauvery delta zone of Tamil Nadu

\begin{tabular}{|c|c|c|}
\hline District & Equation & $\begin{array}{c}\text { Forecast yield, 2018 } \\
\text { (kg/ha) }\end{array}$ \\
\hline Thanjavur & $\mathrm{Y}=6284.1+150.4(\mathrm{Z} 11)$ & 4239 \\
\hline Thiruvarur & $\mathrm{Y}=5722.4+5.7(\mathrm{Z} 121)+26.4(\mathrm{Z} 51)$ & 4939 \\
\hline Nagapattinam & $\mathrm{Y}=2761.9+5.67(\mathrm{Z} 240)+1.0494(\mathrm{Z} 230)$ & 4832 \\
\hline Trichy & $\mathrm{Y}=4512.4+1.7(\mathrm{Z} 120)+0.4(\mathrm{Z} 51)$ & 4521 \\
\hline Karur & $\mathrm{Y}=6882.4+2.7(\mathrm{Z} 130)+17.4(\mathrm{Z} 51)$ & 3886 \\
\hline Perambalur & $\mathrm{Y}=4522.4+15.7(\mathrm{Z} 241)$ & 3396 \\
\hline Ariyalur & $\mathrm{Y}=3422.4+2.7(\mathrm{Z} 51)+4.6(\mathrm{Z} 350)$ & 4437 \\
\hline Cuddalore & $\mathrm{Y}=5121.4+2.9(\mathrm{Z} 241)+14.4(\mathrm{Z} 51)$ & 4505 \\
\hline
\end{tabular}


Table.7 Verification of Rabi, 2017-18 yield forecast

\begin{tabular}{|c|c|c|c|c|c|}
\hline Districts & Area (ha) & $\begin{array}{c}\text { Predicted } \\
\text { yield (F3) } \\
\text { (kg/ha) }\end{array}$ & $\begin{array}{c}\text { Actual } \\
\text { yield } \\
\text { (kg/ha) }\end{array}$ & $\begin{array}{c}\text { Yield } \\
\text { difference } \\
\text { (kg/ha) }\end{array}$ & $\begin{array}{c}\text { Error } \\
(\%)\end{array}$ \\
\hline Thanjavur & 106077 & 4239 & 4010 & 229 & $\mathbf{5 . 7 1}$ \\
\hline Thiruvarur & 128412 & 4939 & 4468 & 471 & $\mathbf{1 0 . 5 4}$ \\
\hline Nagapattinam & 113537 & 4832 & 4047 & 785 & $\mathbf{1 9 . 4 0}$ \\
\hline Trichy & 45780 & 4521 & 4659 & -138 & $\mathbf{- 2 . 9 6}$ \\
\hline Karur & 12313 & 3886 & 4538 & -652 & $\mathbf{- 1 4 . 3 7}$ \\
\hline Perambalur & 22997 & 3396 & 3887 & -491 & $\mathbf{- 1 2 . 6 3}$ \\
\hline Ariyalur & 7167 & 4437 & 3958 & 479 & $\mathbf{1 2 . 1 0}$ \\
\hline Cuddalore & $\mathbf{5 9 4 3 4}$ & $\mathbf{4 5 0 5}$ & $\mathbf{3 9 3 5}$ & $\mathbf{5 7 0}$ & $\mathbf{1 4 . 4 9}$ \\
\hline
\end{tabular}

\section{Statistical Crop Yield Forecasting}

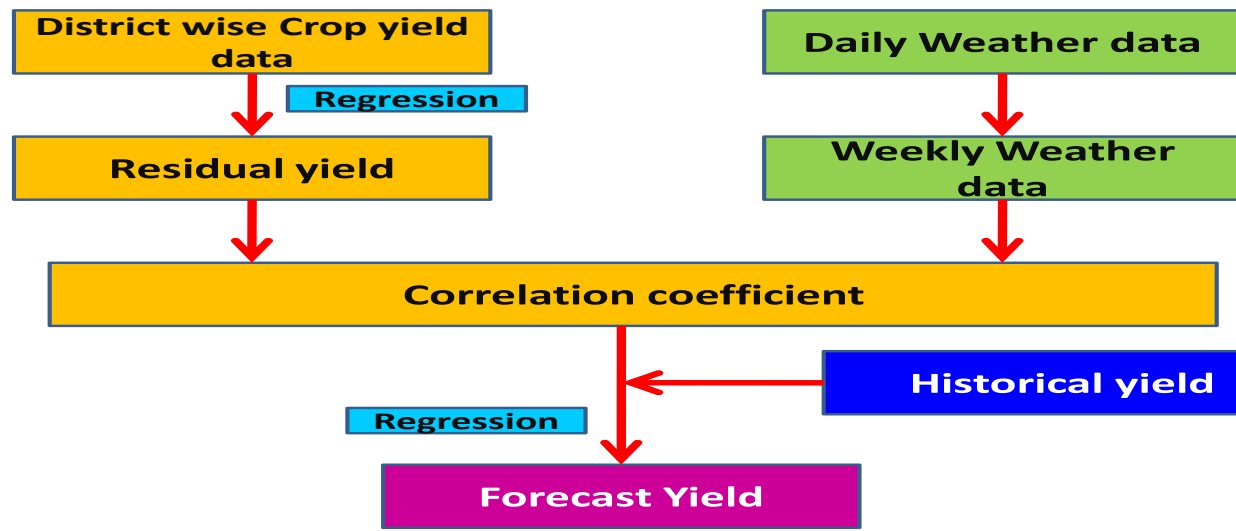

Fig.1 Growth stages of Kharif Rice

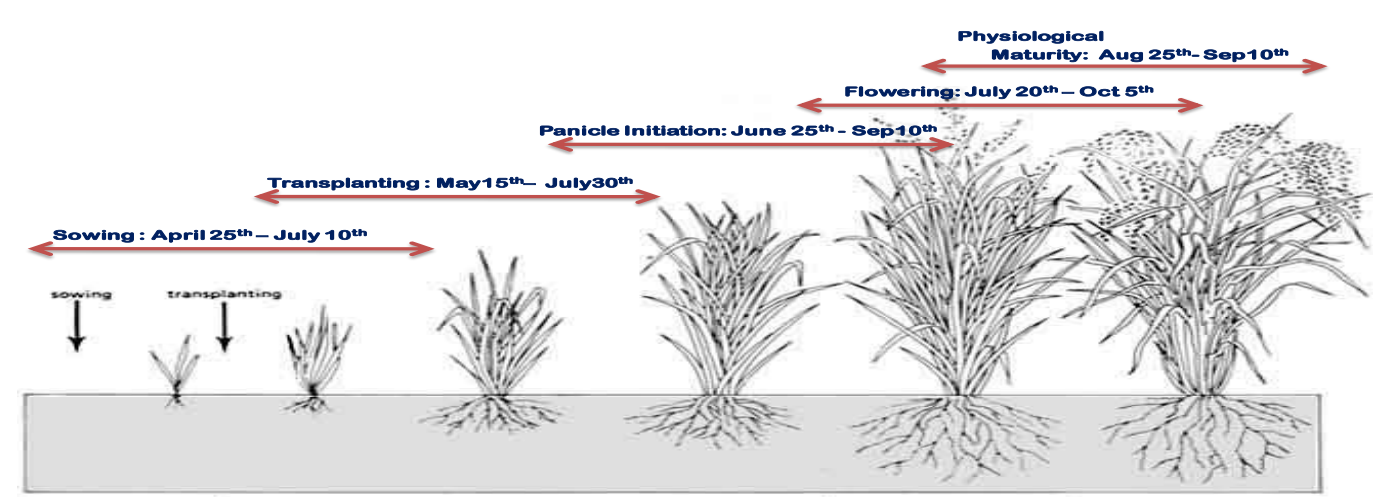


(a)

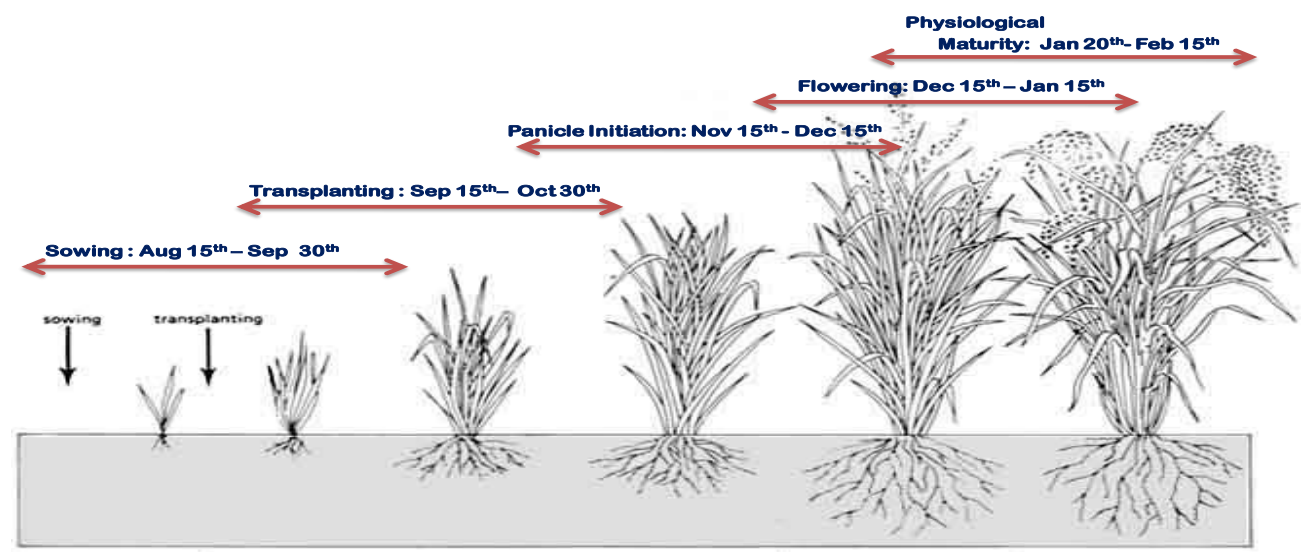

(b)

Fig.2 Growth stages of Rabi Rice

\section{Verification of yield forecast Kharif, 2017}

Verification of yield forecast by using actual yield indicated that actual yield was less than that of predicted yield in all districts. The error percentage varied from -9.60 to 9.14 . The lacunae in the statistical model is that it is taking into consideration of weather parameters prevailed during the season only and not the management practices adopted by the farmers.

\section{Rice yield prediction during Rabi, 2017-18}

\section{Mid-season forecast (F2)}

District wise yield forecast for rice was given for 8 districts (Thanjavur, Thiruvarur, Trichy, Nagapattinam, Cuddalore, Karur, Perambalur and Ariyalur districts) during Rabi, 2016-17 for rice crop. The highest paddy yield of 4556 $\mathrm{kg} / \mathrm{ha}$ was predicted for Trichy district. The major rice varieties during the Rabi season were CR1009, ADT 46, and BPT 5204.

\section{Verification}

Verification of yield forecast by using actual yield indicated that actual yield was less than predicted yield in Thanjavur, Thiruvarur, Nagapattinam, Ariyalur and Cuddaloredistricts. Actual yield was more than predicted yield in Trichy, Karur and Perambalur districts. The error percentage was very less in Trichy (-2.96). Nagapattinam and Cuddalore districts recorded the highest error percentage of 19.40, 14.49, respectively.

\section{References}

Agrawal, Ranjana and Mehta, S.C. (2007). Weather based forecasting of crop yields, pests and diseases - IASRI models. Ind. J. Agri. Sci., 71(7): 487490.

Boltan, F.E. (1981).Optimizing the use of water and nitrogen through soil and crop management. Plant Soil., 58: 231247.

Jayantha Sarkar, (2003). Forecasting rice and wheat yield over different meterological sub-divisions of India using statistical models. J. Ind.Soc.Ag.Stat. 56(1): 8889.

Surrender paul, Chander shekar and S.C. Bhan. (2012). Cotton yield prediction 
for Punjab using weather based statsistical models. Journal of Agrometeorology. 14(2): 184-186.

Tripathi, M.K., B. Mehra, N. Chattopadhyay and K.K. Singh. (2012). Yield prediction of sugarcane and paddy for districts of Uttar Pradesh. Journal of Agrometeorology. 14(2): 173-175.

\section{How to cite this article:}

Senthil Kumar. G. and Subrahmaniyan. K. 2020. Rice Yield Prediction for Cauvery Delta Zone of Tamil Nadu using Weather Based Statistical Model. Int.J.Curr.Microbiol.App.Sci. 9(06): 400-406. doi: https://doi.org/10.20546/ijcmas.2020.906.052 